\title{
Separating Physical Components from Galaxy Spectra by Subspace Methods
}

\section{Ching-Wa Yip ${ }^{1} \dagger$, Alex S. Szalay ${ }^{1}$, Andrew J. Connolly ${ }^{2}$ and Tamas Budavári ${ }^{1}$}

${ }^{1}$ Department of Physics and Astronomy, The Johns Hopkins University, Baltimore, MD 21218, USA

${ }^{2}$ Department of Physics and Astronomy, University of Pittsburgh, Pittsburgh, PA 15260, USA

\begin{abstract}
Using subspace methods, we study the distribution of physical components of galaxies in wavelength space. We find that it is valid to assume that the stellar and the gaseous components of galaxies span complementary subspaces. To first order, stellar and gaseous spectral features can be extracted from galaxy spectra through a simple matrix multiplication. By comparing the stellar continua obtained respectively using the model-based and the empirical approach through a commonality measure, we conclude that the latter may lose higher-order spectral features.
\end{abstract}

Keywords. galaxies: fundamental parameters - techniques: spectroscopic - methods: statistical

\section{Introduction}

A common method of estimating physical parameters of galaxies is by least-square fitting stellar population synthesis models to their spectra. The approach is time-consuming when the model becomes large. This hinders the examination of all possible parameters defining the models (for example, PÉGASE (Fioc \& Rocca-Volmerange 1997) is defined by as much as $\sim 10$ parameters), especially for large sky surveys such as the SDSS which will produce 1 million galaxy spectra. On the other hand, principal components (PCs) are known to be powerful in classifying galaxy spectra (Connolly et al. 1995). This suggests that, as PCs form one kind of basis vectors, a subspace is a good description to the higher dimensional spectral space. In this work, we exploit the subspace methods in extracting physical information from galaxy spectra.

\section{Stellar and Gaseous Subspaces}

The projection matrix for a set of astronomical spectra (Yip et al. 2004) is defined to be $P \equiv \sum_{e}|e\rangle\langle e|$, where $e$ are the eigenspectra (Connolly et al. 1995) of that set of spectra. We construct a projection matrix using the Bruzual \& Charlot (2003). The subspace defined by this projection matrix, $P$ (star), is referred as the stellar subspace. Each galaxy spectrum $f$ in our sample (taken to be various types of SDSS galaxy spectra) is projected onto this stellar subspace, so that the resultant vector, $f($ star), is

$$
f(\text { star }) \equiv P(\text { star }) \times f,
$$

where $\times$ means a matrix multiplication. If the gaseous and stellar subspaces are complementary, we have

$$
P(\text { gas }) \equiv I-P(\text { star }),
$$

$\dagger$ Keck Fellow. Email to authors: cwyip@pha.jhu.edu; szalay@pha.jhu.edu 


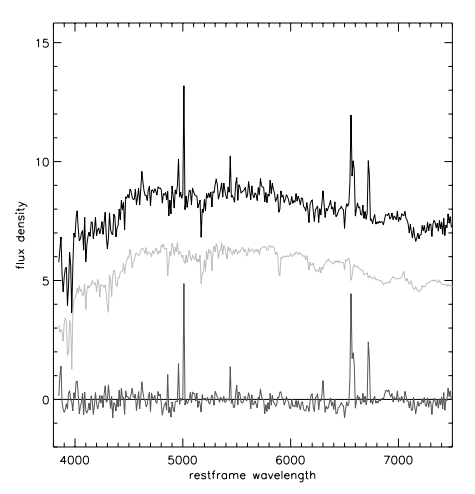

Figure 1. The stellar (light gray) and gaseous (dark gray) components of the observed galaxy spectrum (black) obtained by the subspace method. The flux density of the stellar component is arbitrarily offset for clarity.

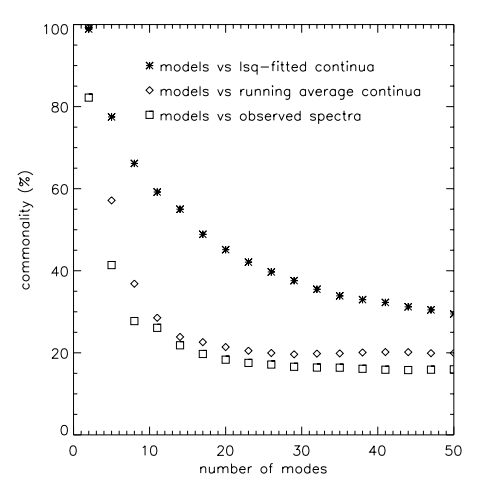

Figure 2. The commonality between the stellar subspaces obtained by different methods, as a function of the number of eigenspectra in each and all of the sets of spectra.

where $I$ is the identity matrix. The gaseous component can be obtained directly by $f($ gas $) \equiv P($ gas $) \times f$.

Fig. 1 shows the resultant projected spectra $f$ (star) and $f$ (gas) for an example galaxy spectrum, which resemble the expected stellar and gaseous contributions. Since for a given subspace, its projection matrix is uniquely defined (Oja 1983), only a single projection matrix is needed to define an arbitrarily complex model.

\section{Definition of Stellar Subspace: Model-Based vs. Empirical}

As we construct the stellar subspace by adopting the stellar population synthesis models, it is important to understand how well this model-based approach is when compared with other approach such as the empirical approach. Our chosen empirical approach is for a particular pixel by averaging the flux between the 40th and 60th quantiles of the flux distribution taken from a wavelength range of $\pm 200 \mathrm{~km} / \mathrm{s}$. The emission lines are masked around $\lambda_{0} \pm 2000 \mathrm{~km} / \mathrm{s}$, with $\lambda_{0}$ being the wavelength of the line center.

To measure the overlap between the stellar subspaces defined by different approaches, we use the commonality measure (see Yip et al. 2004 for its application). Fig. 2 shows that the least-square fit stellar continua (i.e. model-based) do not overlap $100 \%$ with the model spectra because of the sample variation arise from the selection of the galaxy. This will be our null measure, as such no other stellar continuum estimation method would exceed this line. The commonality between the empirical running-average approach for the stellar continua and the model drops below that of the null measure with the number of modes, indicating the loss of higher-order features in the former.

We acknowledge support from the W. M. Keck Foundation, through a grant given to establish a program of data intensive science at JHU.

\section{References}

Bruzual, G. \& Charlot, S. 2003, MNRAS, 344, 1000

Connolly, A. J., Szalay, A. S., Bershady, M. A. et al. 1995, AJ, 110, 1071

Fioc, M. \& Rocca-Volmerange, B. 1997, A\&SA, 326, 950

Oja E. 1983, Subspace Methods of Pattern Recognition, Research Studies Press.

Yip, C. W., Connolly, A. J., Szalay, A. S. et al. 2004, AJ, 128, 585 